\title{
ON THE UNIFORM ERGODIC THEOREM OF LIN
}

\author{
STUART P. LLOYD
}

\begin{abstract}
Lin has given necessary and sufficient conditions for convergence in the uniform operator topology of $A_{n}=\left(I+T+\cdots+T^{n-1}\right) / n, T$ being a Banach space operator satisfying $\left\|T^{n}\right\| / n \rightarrow 0$. We prove a generalization in which the Cesàro means are replaced by any bounded sequence in the affine hull converging uniformly to invariance. In the case where $T: C_{0}(X) \rightarrow C_{0}(X)$ is a transient Feller operator for noncompact locally compact Hausdorff space $X$, we show that $\left\{A_{n}\right\}$ converges strongly but never uniformly.
\end{abstract}

1. Introduction. The following problem was mentioned by Robert $\mathrm{C}$. Sine in correspondence with the present author several years ago. Suppose a Banach space operator satisfies the conditions of the strong ergodic theorem. When is 1 isolated in the spectrum a sufficient condition for the uniform ergodic theorem? Put another way, when 1 is isolated the resolvent has either a simple pole or else an essential singularity at 1 ; when is the latter case excluded? We obtain here a partial result. Namely, the resolvent of a transient Feller operator on a noncompact locally compact Hausdorff state space has a singularity at 1 which is never a simple pole. The author has not been able to construct an example of such an operator where 1 is isolated in the spectrum.

2. The ergodic theorems of Sine and of Lin. With $\mathscr{B}(\mathcal{X})$ the bounded linear operators on a complex Banach space $\mathscr{X}$, let $T \in \mathscr{B}(\mathcal{X})$ be given with spectral radius $r(T)=1$. We abbreviate [uniform] [strong] operator topology to [UOT] [SOT]. Let $\mathscr{P}_{1}$ consist of those $P \in \mathscr{B}(\mathcal{X})$ which can be expressed as $P=$ $\sum_{j=0}^{\infty} a_{j} T^{j}$ where $\sum_{0}^{\infty} a_{j} z^{j}$ has a radius of convergence greater than 1 and $\sum_{0}^{\infty} a_{j}=1$. It will always be assumed that $T$ satisfies condition UI:

(UI) For some $1 \leqslant M_{0}<\infty$ the UOT closure of the set $\left\{(I-T) P: P \in \mathscr{P}_{1}\right.$ and $\left.\|P\| \leqslant M_{0}\right\}$ contains $0 \in \mathscr{B}(\mathcal{X})$.

That is, $\mathscr{P}_{1}$ contains bounded sequences $\left\{P_{n}\right\}$ converging uniformly to invariance: $\lim _{n}\left\|P_{n}-T P_{n}\right\|=0$. We call such a sequence a UI sequence.

Let $\mathcal{L}=\{x \in \mathcal{X}: T x=x\}$ be the invariant vectors of $T$, and let $\mathcal{Q}^{*}$ be the invariant vectors of the adjoint $T^{*} \in \mathscr{B}\left(\mathfrak{X}^{*}\right)$. The generalized Sine Ergodic Theorem proved in [3] asserts that when UI holds the following conditions are equivalent:

(i) 2 separates $2^{*}$;

Received by the editors November 21, 1980 and, in revised form, April 7, 1981.

1980 Mathematics Subject Classification. Primary 47A35.

Key words and phrases. Uniform ergodic theorem. 
(ii) The SOT closure of $\mathscr{P}_{1}$ contains a projection $Q$ onto $\mathcal{Q}$, necessarily unique, and each UI sequence is SOT convergent to $Q$.

(See [4] for a simplified proof and additional references.) After this, " $Q$ exists" will mean that (ii) holds. The case $Q=0$ is included; it requires $2^{*}=0$ as well as $2=0$.

The spectrum of $T \in \mathscr{B}(\mathscr{X})$ is denoted by $\sigma(T)$ or $\sigma(T ; \mathscr{X})$, the resolvent set by $\rho(T)$, the range by $\Re(T)$. The resolvent $R_{\lambda}(T)=(\lambda I-T)^{-1}, \lambda \in \rho(T)$, can be expressed as $R_{\lambda}(T)=\sum_{j=0}^{\infty} \lambda^{-j-1} T^{j}$ when $|\lambda|>1$; we define $P_{\lambda}(T)=$ $(\lambda-1) R_{\lambda}(T), \lambda \in \rho(T)$, and have $P_{\lambda}(T) \in \mathscr{P}_{1}$ when $|\lambda|>1$.

The following result is phrased to address the Sine problem of $\S 1$. Some parts of the proof are straightforward generalizations of Lin's methods of [2], although we use also the operator calculus and spectral considerations [1, Chapter VII]. The assumption that $Q$ exists simplifies most of the arguments. The subspaces $2=$ $\Re(Q)$ and $R(I-Q)$ are invariant under $T$ and $I-T$, since $Q T=T Q$.

Theorem 1. Let $T \in \mathscr{B}(X)$ of spectral radius 1 be such that $U I$ holds and $Q$ exists. Then the following conditions are equivalent.

[i] $R_{\lambda}(T)-Q /(\lambda-1)$ is analytic at $\lambda=1$;

[ii] [iii] For [some] [each] sequence $\lambda_{n} \downarrow 1,\left\{P_{\lambda_{n}}(T)\right\}$ is UI and is UOT convergent to $Q$;

[iv] [v] [Some] [Each] UI sequence $\left\{P_{n}\right\}$ is UOT convergent to $Q$;

[vi] Some $P \in \mathscr{P}_{1}$ satisfies $\|P-Q\|<1$;

[vii] $\Re(I-T)$ is closed;

[viii] As an operator in $\Re(\Re(I-Q))$, the restriction $T \mid \Re(I-Q)$ has spectrum $\sigma(T ; \Re(I-Q))$ which does not contain 1 .

Proof. [vii] $\Leftrightarrow$ [viii]. On $2, I-T$ vanishes. On $\Re(I-Q), I-T$ is $1-1$, since the nullspace of $I-T$ is 2 and $\mathcal{X} / \mathcal{Q} \approx \mathscr{R}(I-Q)$. Thus a left inverse $\Theta$ exists, defined on $R(I-T)$, satisfying $\Theta(I-T)=I-Q$; the operator $\Theta$ is linear but need not be bounded. In fact, $\Theta$ is bounded iff $\Re(I-T)$ is closed in $\mathcal{X}$ [1, Theorem II.2.2]. It is known that $\Re(I-T)$ is dense in $R(I-Q)$ when $Q$ exists; see [4]. Thus $\mathscr{R}(I-T)=\mathscr{R}(I-Q)$ iff $R(I-T)$ is closed, and in this event $\Theta \in \Re(X)$ exists and is determined by $\Theta(I-T)=(I-T) \Theta=I-Q, \Theta Q=$ $Q \Theta=0$. On the other hand, the restriction $(I-T) \mid \Re(I-Q)$ has a bounded inverse in the Banach space $\Re(I-Q)$ iff $0 \notin \sigma(I-T ; R(I-Q))$ iff $1 \notin$ $\sigma(T ; \Re(I-Q))$.

[viii] $\Leftrightarrow\left[\right.$ i]. From $(\lambda I-T) Q=Q(\lambda I-T)=(\lambda-1) Q$ follows $R_{\lambda}(T) Q=$ $Q R_{\lambda}(T)=Q /(\lambda-1)$, whence

$$
R_{\lambda}(T)(I-Q)=(I-Q) R_{\lambda}(T)=R_{\lambda}(T)-Q /(\lambda-1), \quad \lambda \in \rho(T) .
$$

The operator $R_{\lambda}(T)(I-Q)$ vanishes on 2 ; its restriction to $\mathcal{R}(I-Q)$ can be regarded as the resolvent $R_{\lambda-1}(T-I ; \mathcal{R}(I-Q))$, since $(T-I)(I-Q)=$ $(I-Q)(T-I)=T-I$ in $\mathscr{B}(\mathscr{X})$. This resolvent is analytic at $\lambda-1=0$ iff $0 \notin$ $\sigma(T-I ; \Re(I-Q))$ iff $1 \notin \sigma(T ; \Re(I-Q))$.

[i] $\Rightarrow$ [iii]. If $\left\{P_{n}\right\}$ is UOT convergent to $Q$ then $\left\{P_{n}\right\}$ is necessarily UI, since $\left\{P_{n}\right\}$ is bounded and $\left\|(I-T) P_{n}\right\|=\left\|(I-T)\left(P_{n}-Q\right)\right\| \leqslant\|I-T\|\left\|P_{n}-Q\right\|$. 
[vii] $\Rightarrow[v]$. With $\Theta$ above bounded,

$$
\left\|P_{n}-Q\right\|=\left\|(I-Q) P_{n}\right\|=\left\|\Theta(I-T) P_{n}\right\|<\|\Theta\|\left\|(I-T) P_{n}\right\| .
$$

[vi] $\Rightarrow$ [vii] (Lin). If $\|P-Q\|=\|P(I-Q)\|<1$ then $(I-P) \mid R(I-Q)$ is invertible in $\mathscr{B}(\mathscr{R}(I-Q))$; determine $\gamma \in \mathscr{B}(\mathcal{X})$ by $\gamma(I-P)=(I-P) \gamma=I$ $-Q, \gamma Q=Q \gamma=0$. With $P=f(T) \in \mathscr{P}_{1}$, define $g(z)$ by

$$
g(z)=[f(z)-1] /(z-1)
$$

this is analytic on an appropriate neighborhood of $\sigma(T)$, so $g=g(T)$ exists in $\mathscr{B}(\mathcal{X})$ and $g(I-T)=(I-T) g=I-P, g Q=Q g$. It is straightforward that $\gamma g=g \gamma$ is the previously discussed $\Theta \in \mathscr{B}(\mathfrak{X})$.

Since $\sigma(T ; \mathcal{X})=\sigma(T ; \mathcal{Q}) \cup \sigma(T ; \Re(I-Q))$ and $\sigma(T ; \mathcal{Q}) \subset\{1\}$, we have $\sigma(T ; \Re(I-Q)) \supset[\sigma(T) \backslash\{1\}]^{-}$; condition [viii] necessarily fails if 1 is an accumulation point of $\sigma(T)$. Suppose $1 \in \sigma(T)$ is isolated in $\sigma(T)$, and let $E=$ $(2 \pi i)^{-1} \int_{C} R_{\lambda}(T) d \lambda$ be the corresponding spectral projection; the contour is a small circle in $\rho(T)$ with 1 interior and $\sigma(T) \backslash\{1\}$ exterior. From $R_{\lambda}(T) Q=Q R_{\lambda}(T)=$ $Q /(\lambda-1)$ follows $E Q=Q E=Q$. Let $\Delta=E-Q$ denote the complementary part: $\Delta^{2}=\Delta, \Delta E=E \Delta=\Delta, \Delta Q=Q \Delta=0$. If $\Delta \neq 0$ define $N \in \mathscr{B}(\mathcal{X})$ by $T \Delta=\Delta+N$. Then $N \Delta=\Delta N=N$ and $\sigma(N ; \mathfrak{X})=\{0\}$. Since $N$ is quasinilpotent,

$$
R_{\lambda}(T) \Delta=\frac{\Delta}{(\lambda-1) I-N}=\frac{\Delta}{\lambda-1}+\frac{N}{(\lambda-1)^{2}}+\frac{N^{2}}{(\lambda-1)^{3}}+\cdots, \quad \lambda \neq 1,
$$

is entire in $1 /(\lambda-1)$. Furthermore, $N x \neq 0$ if $0 \neq x \in R(\Delta)$; otherwise $T(\Delta x)=$ $(\Delta x)$ would imply $Q x=x$, contradicting $Q \Delta=0$. It is obvious from this that $N^{j} x \neq 0$ if $0 \neq x \in \Re(\Delta)$ for each $j \geqslant 0$. It follows that $R_{\lambda}(T)$ has an essential singularity at $\lambda=1$ if $\Delta \neq 0$, and Sine's problem is: characterize the case $\Delta=0$.

3. A class of counterexamples. We show that condition [viii] of Theorem 1 fails in an interesting class of examples. Let $X$ be a noncompact locally compact Hausdorff space, and let $C_{0}(X)$ be the continuous complex functions vanishing at infinity, with the usual supremum norm. We identify the bounded Radon measures $\mathscr{R}(X)$ as the Banach space conjugate of $C_{0}(X)$, and denote by $\mathfrak{R}^{+}(X)$ the elements $\nu \geqslant 0$.

Let $T: C_{0}(X) \rightarrow C_{0}(X)$ be a Feller operator on $C_{0}(X)$. By this we mean

$$
(T f)(x)=\int f\left(x^{\prime}\right) t_{x}\left(d x^{\prime}\right), \quad x \in X, f \in C_{0}(X),
$$

where the representing kernel $t: X \rightarrow \mathfrak{R}(X)$ is weakly* continuous and has the properties $t_{x} \geqslant 0$ and $t_{x}(X)=1, x \in X$, and $\lim _{x \rightarrow \infty} t_{x}(K)=0$ for any compact set $K \subset X$. The adjoint $T^{*}: \mathfrak{T}(X) \rightarrow \mathfrak{T}(X)$ has representation

$$
\left(T^{*} \nu\right)(A)=\int_{X} t_{x}(A) \nu(d x), \quad \text { Borel } A \subset X, \nu \in \mathscr{T}(X)
$$

We note that $\|\nu\|=\nu(X)$ when $\nu \in \mathfrak{R}^{+}(X)$, so $\left\|T^{*} \nu\right\|=\|\nu\|, \nu \in \mathfrak{T}^{+}(X)$; this implies $\|T\|=1$. 
We assume that the action of $T$ is either null recurrent or transient:

$$
\lim _{N \rightarrow \infty} \sup (1 / N) \sum_{n=0}^{N-1}\left(T^{* n} \nu\right)(K)=0,
$$

$$
\text { each compact subset } K \subset X, \nu \in M^{+}(X) \text {. }
$$

To be a little more explicit, each compact subset $K$ of the state space $X$ is either null recurrent or transient for the discrete parameter Markov process which has $\nu /\|\nu\|$ as initial probability and $t$ as transition probability.

THEOREM 2. Let $T: C_{0}(X) \rightarrow C_{0}(X)$ be a null recurrent or transient Feller operator on noncompact locally compact Hausdorff space $X$. Then UI holds, and $Q$ exists with $Q=0$. However, $\sigma(T) \ni 1$, and the resolvent $R_{\lambda}(T)$ cannot have a simple pole at $\lambda=1$.

Proof. Each $T^{j}: C_{0}(X) \rightarrow C_{0}(X)$ is a Feller operator, with representing kernel $t^{(j)}, j \geqslant 0$. Since $\left\|T^{j}\right\|=1$, the usual $A_{n}=(1 / n) \sum_{j=0}^{n-1} T^{j}, n \geqslant 1$, constitute a UI sequence. Let us first prove $\mathcal{L}=0$. The modulus of any $f \in C_{0}(X)$ attains $\|f\|$ on $X$, since $f$ vanishes at infinity. Suppose $0 \neq f \in 2$, let $x_{0} \in X$ be such that $\left|f\left(x_{0}\right)\right|=\|f\|$, and define a nonempty compact set $K$ by $K=\{x \in X: f(x)=$ $\left.f\left(x_{0}\right)\right\}$. From $T^{j} f=f$ and $t^{(j)} \geqslant 0, t^{(j)}(X)=1$, we obtain $t_{x}^{(j)}(K)=1, x \in K, j \geqslant 0$. This contradicts (1), proving $2=0$.

We show next that $2^{*}=0$. If $0 \neq \nu \in 2^{*}$, the real and imaginary parts of $\nu$ are separately invariant, since $T^{*}$ is real. Thus we may assume that $0 \neq \nu \in 2^{*}$ is real. Then $0 \neq|\nu| \in 2^{*}$, by the well-known argument $T^{*}|\nu| \geqslant|\nu|,\left\|T^{*}|\nu|\right\|=\||\nu|\|$. Suppose a compact set $K$ is such that $|\nu|(K)>0$. Then $\left(T^{* n}|\nu|\right)(K)=|\nu|(K)>0$ contradicts (1), showing $2^{*}=0$. By the Sine Ergodic Theorem, $Q$ exists; moreover, $Q=0$.

It remains to show that $\sigma(T) \ni 1$. The element $\mathscr{G}$ of the conjugate Banach space $\mathfrak{R} *(X)$ is determined by

$$
(\nu, \mathscr{G})=\nu(X), \quad \nu \in \mathscr{R}(X) .
$$

From $(\nu, G)=\|\nu\|, \nu \in \Re^{+}(X)$, follows $G \neq 0$. The condition $t_{x}(X)=1, x \in X$, implies $\left(T^{*} \nu, \mathscr{G}\right)=(\nu, \mathscr{G}), \nu \in \mathfrak{N}(X)$, and hence $T^{* *} \mathscr{G}=\mathscr{G}$, where $T^{* *}: \mathfrak{T}^{*}(X)$ $\rightarrow \mathscr{T}^{*}(X)$ is the adjoint of $T^{*}$. Since $\mathcal{G} \neq 0$, it belongs to the point spectrum at $\lambda=1$, i.e., $\sigma\left(T^{* *}\right) \ni$ 1. But $\sigma\left(T^{* *}\right)=\sigma(T)$ by [1, Lemma VII.3.7].

Theorem 2 is applicable in a more general setting. We are not concerned with boundary theory here, so we present matters without proof. We confine our remarks to the transient case. Suppose the space $X$ above is open and dense in a compact Hausdorff space $Y$, and that the boundary $M=Y \backslash X$ is regular; the following conditions are supposed to be satisfied. First, the property

$$
\lim _{\substack{x \rightarrow y \\ x \in X}} t_{x}=\delta_{y} \quad \text { weakly* in } \Re(Y), \quad y \in M,
$$

where $\delta_{y}$ is the unit point measure at $y$, extends the given operator $T: C_{0}(X) \rightarrow$ $C_{0}(X)$ to a Feller operator $\check{T}: C(Y) \rightarrow C(Y)$. Also, $\grave{Q}$ exists for $\check{T}$, the Choquet 
boundary of $\breve{\mathscr{Q}}$ is $M$, and $\breve{\mathscr{Q}}$ is isometrically isomorphic to $C(M)$. Then $\Re(I-\check{Q})$ $=\{f \in C(Y): f(M)=0\}$, and this is isometrically isomorphic to $C_{0}(X)$. By Theorem $2, \sigma(\check{T} ; \mathcal{R}(I-\check{Q})) \ni 1$, so the uniform ergodic theorem fails for any such $\check{T}$.

\section{REFERENCES}

1. Nelson Dunford and Jacob T. Schwartz, Linear operators. I, Interscience, New York, 1958.

2. Michael Lin, On the uniform ergodic theorem, Proc. Amer. Math. Soc. 43 (1974), 337-340.

3. Stuart P. Lloyd, On the mean ergodic theorem of Sine, Proc. Amer. Math. Soc. 56 (1976), 121-126.

4. Ryotaro Sato, The Hahn-Banach theorem implies Sine's mean ergodic theorem, Proc. Amer. Math. Soc. 77 (1979), 426.

Bell Laboratories, Whippany, NeW Jersey 07981 